\title{
Development of a Bacillus subtilis expression system using the improved Pglv promoter
}

\author{
Yang M Ming ${ }^{* \dagger}$, Zhang W Wei ${ }^{\dagger}$, Chen Y Lin and Gong Y Sheng
}

\begin{abstract}
Background: $B$. subtilis is an important organism in the biotechnological application. The efficient expression system is desirable in production of recombinant gene products in B. subtilis. Recently, we developed a new inducible expression system in B. subtilis, which directed by B. subtilis maltose utilization operon promoter $P_{g / v}$. The system demonstrated high-level expression for target proteins in $B$. subtilis when induced by maltose. However, the system was markedly repressed by glucose. This limited the application of the system as a high-expression tool in biotechnology field. The aim of this study was to further improve the $P_{\text {glv }}$ promoter system and enhance its expression strength.
\end{abstract}

Results: Here, site-directed mutagenesis was facilitated to enhance the expression strength of $\mathrm{P}_{g / v}$. The transcription level from four mutants was increased. Production of $\beta$-Gal from the mutants reached the maximum 1.8 times as high as that of wildtype promoter. When induced by $5 \%$ maltose, the production of $\beta$-Gal from two mutants reached 14.3 $\mathrm{U} / \mathrm{ml}$ and $13.8 \mathrm{U} / \mathrm{ml}, 63.5 \%$ and $57.5 \%$ higher than wildtype promoter $(8.8 \mathrm{U} / \mathrm{ml})$ respectively. Thus, site-directed mutagenesis alleviated the repression of glucose and improved the expression activity. To further improve the promoter system, the B. subtilis expression host was reconstructed, in which B. subtilis well-characterized constitutive promoter P43 replaced the promoter of the glv operon in B. subtilis chromosome through a double crossover event. The $\beta$-galactosidase production from the improved system $(21.1 \mathrm{U} / \mathrm{mL})$ increased compared to that from origin system. Meanwhile, the repression caused by glucose was further alleviated.

Conclusions: In this study, we obtained a mutated promoter Pg/v-M1 through site-directed mutagenesis, which demonstrated high expression strength and alleviated the repression caused by glucose. Moreover, we alleviated the repression and enhanced the expression activity of the Pg/v-M1 promoter system via reconstruction of the B. subtilis host. Thus, we provided a valuable expression system in B. subtilis.

\section{Background}

B. subtilis is an important organism in the biotechnological application, regarding its non-pathogenic and wellcharacterized biochemical and physiological property $[1,2]$. The genetic engineering of $B$. subtilis played a significant role in biotechnological application and industry $[3,4]$. A controllable expression system is desirable in efficient production of recombinant gene products in B. subtilis [5-7]. The most prominently and widely used induction systems in B. subtilis are mediated by promoters $\mathrm{P}_{\text {spac }}$ and $\mathrm{P}_{x y l}$. The disadvantage of these systems is that the inducer is costly for industrial application [8-10].

\footnotetext{
*Correspondence: yangjinxin@vip.163.com

1 College of Animal Science and Technology, Northwest A\&F University,

Yangling 712100, China

+ Contributed equally

Full list of author information is available at the end of the article
}

Recently, we developed a new inducible expression system in B. subtilis [11], which directed by B. subtilis maltose utilization operon promoter $\mathrm{P}_{g l v}[12,13]$. The system demonstrated high-level expression for target proteins in B. subtilis when induced by maltose. Moreover, cheap and safe inducer makes the system a potential promoter system in industrial application. However, the system was markedly repressed by glucose, in which the glucose repressed the $\mathrm{P}_{g l v}$ promoter via a catabolism repression element (cre) located downstream of the transcription origin site of the $\mathrm{P}_{g l v}$ promoter [11-13]. This limited the application of the system as a high-expression tool in biotechnology field. To improve the $\mathrm{P}_{g l v}$ promoter system, site-directed mutagenesis of several nucleotides downstream the transcription origin site of $\mathrm{P}_{g l v}$ was facilitated via overlap polymerase chain reaction (PCR) in vitro in 
this study. To further alleviate the repression and enhance the expression strength of the $\mathrm{P}_{g l v}$ promoter, the B. subtilis expression host was reconstructed, in which the constitutive promoter $\mathrm{P} 43$ replaced the promoter of the $g l v$ operon in the B. subtilis chromosome through a double crossover event.

\section{Results and discussion}

Site-direct mutagenesis of Pglv promoter and examination of the expression strength

In the mutant $P_{g l v}-\mathrm{M} 1$, two bases located at the conservation sequence of catabolism repression element were mutated (GCTAT). According to the 3' sequence of $16 \mathrm{~s}$ RNA, the ribosome binding site was optimized in the mutants $P_{g l v}-\mathrm{M} 2$ and $P_{g l v}-\mathrm{M} 3$, based on $P_{g l v}-\mathrm{M} 1$, Additionally, based on $P_{g l v}-\mathrm{M} 3$, the two bases downstream Shine-Dalgarno sequence (SD sequence) of the $P_{g l v}$ promoter was mutated, resulting in $P_{g l v}$-M4.

There is a typical conservation sequence of catabolism repression element (cre), overlapping with the ribosome binding site, downstream of the transcription origin site of the $\mathrm{P}_{g l v}$ promoter $[11,13]$. In order to alleviate the repression of glucose and improve the expression strength of the $\mathrm{P}_{g l v}$ promoter, the site-directed mutagenesis of cre sequence is performed. To further enhance the expression of gene downstream the promoter, we try to improve the ribosome-binding site sequence. According to the sequence of hydroxyl end of $16 \mathrm{~S}$ rRNA, the nucleotides downstream the SD sequence were select to modify in pJRINM4.

To examine the expression efficiency of the obtained four mutants, they were sub-cloned and engineered with synthetic ribosome binding site. The resultant recombinants pJRINM1, pJRINM2, pJRINM3 and pJRINM4, in which the $\operatorname{bgaB}$ was under the control of four mutants respectively, were transformed into $B$. subtilis 1 A747 to investigate the expression of $\beta$-Gal. Real-time PCR assay (Figure 1A) showed that compared with the pLJ-7, the transcription amount from the pJRINM1, pJRINM2 and pJRINM3 were increased in different degrees, in which the mutant pJRINM1 is obviously prior to the pLJ-7. This suggested the site-directed mutagenesis of the Pglv promoter is efficient in these three mutants.

To further verify the efficiency of mutated promoters, the $\beta$-Gal driven by these promoters was determined. The production of $\beta$-Gal from pJRINM1, pJRINM2 and pJRINM3 increased compared with that from pLJ-7. Amongst the production of $\beta$-Gal from pJRINM1, pJRINM2 was 1.8 fold $(1.6 \mathrm{U} / \mathrm{mL})$ and 1.7 fold $(1.5 \mathrm{U} / \mathrm{mL})$ of that from wildtype promoter $(0.9 \mathrm{U} / \mathrm{mL})$ after $24 \mathrm{~h}$ culture, respectively. Whereas, the production of $\beta-$ Gal from pJRINM4 was obviously decreased, about $58.2 \%$ of that from wildtype promoter after $24 \mathrm{~h}$ culture (Figure 1B).
This showed that site-directed mutagenesis in pJRINM4 has negative effect on the $\mathrm{P}_{g l v}$ promoter. We speculated that the three mutated sites including the SD sequnence may change the space structure of the promoter, and the result reduced the transcript level.

While, the growth trend of $B$. subtilis $1 \mathrm{~A} 747$ harbouring pJRINM1, pJRINM2, pJRINM3, pJRINM4 or pLJ-7 was approximately same (Additional file 1). It confirmed that the difference of $\beta$-Gal production was not caused by the cell amount.

To further assay these promoters, we tested the growth curves (Additional file 2) and the $\beta$-Gal activity (Figure $1 C)$ under the induction conditions. When induced with $5 \%$ maltose, the production of $\beta$-Gal from pJRINM1 and pJRINM2 reached $14.3 \mathrm{U} / \mathrm{mL}$ and $13.8 \mathrm{U} / \mathrm{ml}$, up by $63.5 \%$ and $57.5 \%$ compared with wildtype promoter $(8.77 \mathrm{U} / \mathrm{mL})$ at $24 \mathrm{~h}$, respectively. Meanwhile, the $\beta$-Gal production from pJRINM4 was $4.3 \mathrm{U} / \mathrm{mL}$, only $48.8 \%$ of that from wildtype promoter.

SDS-PAGE assay (Figure 1D) demonstrated the $\beta$-Gal production from pJRINM1 and pJRINM2 was obviously higher than that from $\mathrm{pLJ}-7$, further confirming that the two mutants enhanced the expression strength of $\mathrm{P}_{g l v}$ promoter.

\section{Effect of site-directed mutagenesis on the repression caused by glucose}

To examine the effect of mutant on alleviating repression caused by glucose, the four recombinants were cultured in LB medium supplemented with $5 \%$ maltose and 5\% glucose, and the growth curves (Additional file 3 ) and $\beta$ Gal production (Figure 2A) was measured, respectively. The $\beta$-Gal production from wildtype promoter was only $1 / 17$ and $1 / 5$ of that in medium without glucose supplement at $24 \mathrm{~h}$ and $30 \mathrm{~h}$, respectively. Whereas the $\beta$-Gal production from PJRINM1 was $1 / 4$ and $1 / 3$ of that in medium without glucose supplement at $24 \mathrm{~h}$ and $30 \mathrm{~h}$, respectively. While, the $\beta$-Gal production from pJRINM2, pJRINM3 and pJRINM4 was $1 / 6,1 / 6$ and $1 / 4$ of that in medium without glucose supplement at $24 \mathrm{~h}$, respectively. Thus, these mutants alleviated the repression caused by glucose, especially pJRINM1.

Taken together, since the pJRINM1 demonstrated advantage in improvement of expression strength and alleviation of repression caused by glucose, the mutated promoter Pglv-M1 is a good candidate as an expression element in B. subtilis biotechnology application. Subsequently, an effort was made to further probe the relation between glucose and $\beta$-Gal driven by the pJRINM1. When B. subtilis harbouring PJRINM1 was cultured in LB medium with $5 \%$ glucose, reducing sugar assay (Figure 2B) showed that the reducing sugar decreased from $12 \mathrm{~h}$ to $48 \mathrm{~h}$. Additionally, we detected the actual concentration of glucose during the culture. Figure $2 \mathrm{C}$ showed a 


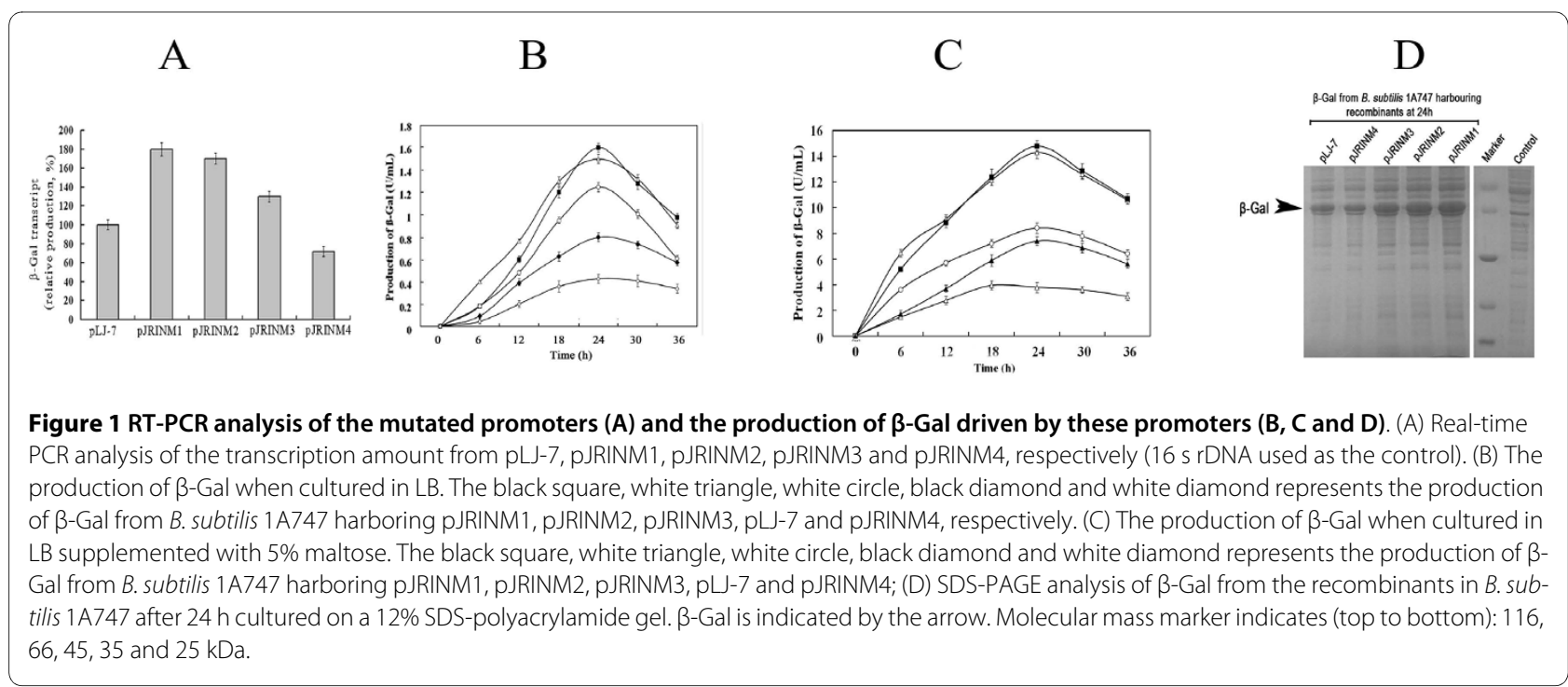

trend that with the decrease of the glucose concentration during cultivation, the $\beta$-Gal production from the recombinant sharply increased in the medium supplemented with $5 \%$ maltose plus $5 \%$ glucose.

\section{Reconstruction of the $B$. subtilis strain to further improve the expression system}

The abovementioned results suggested that the promoter Pglv-M1 alleviated the repression caused by glucose; however, the glucose still exerted repression on the system. We speculated that one of the possible causes of the residual repression was that the maltose utilization operon located in the chromosome of the B. subtilis was nevertheless negatively regulated by glucose, in which GlvR as a positive regulator of the Pglv-M1 promoter system was driven by the operon native promoter $\mathrm{P}_{g l v}$. To further improve the Pglv-M1 promoter system, we replaced maltose utilization operon promoter P $g l v$ with B. subtilis constitutive promoter P43. Southern blot anal- ysis (Figure 3A) and PCR detection indicated that the double crossover event occurred in the resultant strain $B$. subtilis BCYL as expected.

To probe the efficiency of the improved expression system, the mutant pJRINM1 was transformed into BCYL and expression experiments were carried out. Figure $3 \mathrm{~B}$ indicates that, with $5 \%$ maltose induction, $\beta$-Gal production reached a maximum $(21.1 \mathrm{U} / \mathrm{mL})$ at $24 \mathrm{~h}$ from $B$. subtilis BCYL harboring PJRINM1, and this was 50.3\% higher than that from B. subtilis $1 \mathrm{~A} 747$ harboring pIRINM1. For investigating the repression caused by glucose on the improved expression system, BCYL harboring pJRINM1 was cultivated in LB supplemented with $5 \%$ maltose plus $5 \%$ glucose, yielded about 4-fold higher production of $\beta$-Gal than that of B. subtilis $1 \mathrm{~A} 747$ harboring pJRINM1 at $24 \mathrm{~h}$ (Figure 3B). Therefore, the reporter gene production driven by the improved expression system increased obviously when supplemented with $5 \%$ glucose or not.
A

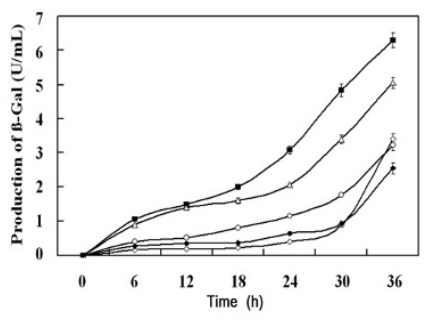

B

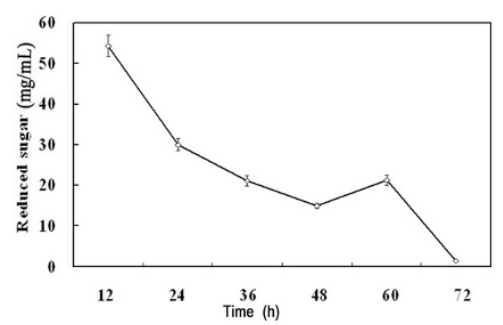

$\mathrm{C}$

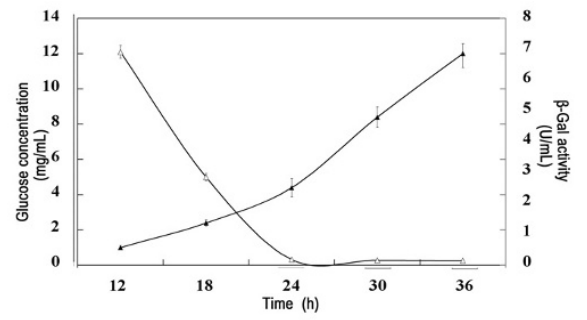

Figure 2 Characterization of the mutated promoters. The production of $\beta$-Gal from B. subtilis 1 A747 harboring mutants supplemented with $5 \%$ maltose plus $5 \%$ glucose. The black square, white triangle, white circle, black diamond and white diamond represents the production of $\beta$-Gal from B. subtilis 1 A747 harboring pJRINM1, pJRINM2, pJRINM3, pLJ-7 and pJRINM4, respectively; (B) Reduced sugar concentration of culture of $B$. subtilis 1 A747 harboring pJRINM1 in LB supplemented with 5\% maltose; (C) Glucose actual concentration and $\beta$-Gal activity detection from B. subtilis 1 A747 harboring pJRINM1 in LB supplemented with 5\% maltose plus 5\% glucose. The black triangle represents the $\beta$-Gal production. The white triangle represents the glucose actual concentration. 

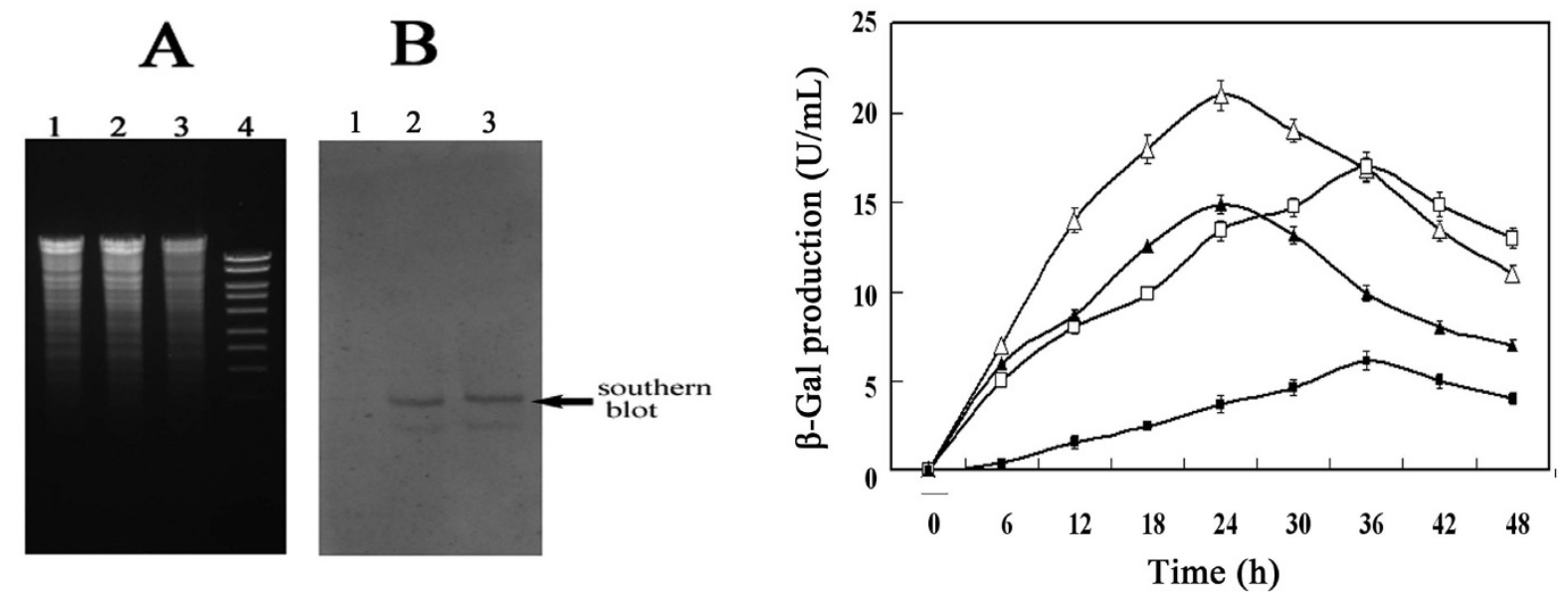

Figure 3 Reconstruction of $B$. subtilis host. (A) Agarose-gel-electrophoresis of chromosome DNA digested with Apal and EcoRl. Lane1, treated chromosome DNA from B. subtilis 1A747 [this served as the negative control]; lanes 2 and 3, treated chromosome DNA from B. subtilis BCYL; lane 4, molecular weigh marker. (B) Southern blot analysis of recombinant B. subtilis BCYL chromosome DNA. The lanes 1, 2 and 3 are that the same as in (A). (C) $\beta$ Gal production by the improved expression system. White triangle and white square represents the $\beta$-Gal production by $B$. subtilis BCYL harboring pJRINM1 cultures in LB with $5 \%$ maltose and LB with $5 \%$ maltose plus $5 \%$ glucose, respectively; black triangle and black square represents the $\beta$-Gal production by $B$. subtilis 1 A747 harboring pJRINM1 when cultured in LB with $5 \%$ maltose and LB with $5 \%$ maltose plus $5 \%$ glucose, respectively.

Controllable and strong promoter is the essential element to achieve high-level expression of target gene in the $B$. subtilis genetic engineering. $B$. subtilis maltose utilization promoter is a potential control element in the biotechnological application; however, glucose as a repressor influenced the strength of $\mathrm{P}_{g l v}$. In the maltoseinducible system, maltose plays two roles as an inductor: on one hand, it positively regulates the transcription of the $\mathrm{P}_{g l v}$ promoter; on the other hand, as a fermentation carbon source, its metabolism production-glucose repressed the activity of promoter. There is a dynamic balance between the two carbon sources during the fermentation. Maltose was a positive regulation factor and, meanwhile degraded as one of carbon sources during this process; in the later stage of fermentation, both the total carbon sources and glucose are at relatively low level. When induced by $5 \%$ maltose, the production of $\beta-G a l$ reached the maximum at $24 \mathrm{~h}$ and then decreased. In the glucose repression experiment, expression strength of both the mutated and wildtype promoter had a drastic increase after cultured for $24 \mathrm{~h}$. This may be the utilization of glucose, as an easy ferment carbon source, was prior to maltose and the amount of glucose in total carbon source declines to a relatively low level in the later stage of fermentation. As a result, the repression caused by glucose was relatively alleviated after $24 \mathrm{~h}$.

\section{Conclusions}

In this study, we obtained a mutated promoter Pglv-M1 through site-directed mutagenesis, which demonstrated high expression strength and alleviated the repression caused by glucose. Moreover, we further alleviate the repression and enhance the expression activity of the Pglv-M1 promoter system via reconstruction of the $B$. subtilis host. Thus, we improved the promoter system and provided a valuable expression system in B. subtilis.

\section{Methods}

\section{Bacterial strains, plasmids and growth conditions}

B. subtilis 1A747 was a generous gift from the Bacillus Genetic Stock Center (BGSC). Escherichia coli DH5a was purchased from Novagen (Darmstadt, Germany). The bacterial strains were cultured in Luria-Bertani (LB) medium at $37^{\circ} \mathrm{C}$. Maltose and glucose were added as required. The following concentrations of antibiotics were used for selection: $100 \mu \mathrm{g} / \mathrm{mL}$ ampicillin (Amp), 5 $\mu \mathrm{g} / \mathrm{mL}$ chloramphenicol $(\mathrm{Cm})$ and $50 \mu \mathrm{g} / \mathrm{mL}$ spectinomycin (Spec). The plasmids used in this study were listed in Additional file 4.

\section{Primers and oligonucleotides}

Polymerase chain reaction (PCR) primers and oligonucleotides used in this study were listed in Additional file 5.

\section{General manipulation}

General recombinant DNA technique was carried out using standard techniques [14]. The transformation of $B$. subtilis was performed by electroporation $[15,16]$.

\section{Site-directed mutagenesis of Pglv promoter via overlap PCR}

The bases downstream the transcription original sites were site-directed mutated by using of overlap PCR. The 
sequences of primers that were used in this experiment are shown in Additional file 5, and the mechanism of the mutagenesis method and the positions of the primers are depicted in Figure 4.

A 360 bp fragment upstream of the maltose promoter and a $300 \mathrm{bp}$ fragment downstream of the maltose promoter waiting for site-directed mutagenesis were ampli- fied from Bacillus subtilis $1 \mathrm{~A} 747$ genome DNA as a template respectively, using primer pairs overlap-1-up/ overlap-1-down and overlap-2-up/overlap-2-down, in which there are $30 \mathrm{bp}$ overlap in the two amplified fragments and the two mutant sites were introduced by primers overlap-1-down and overlap-2-up. With overlap-1-up and overlap-2-down as primers, and the mixture of two

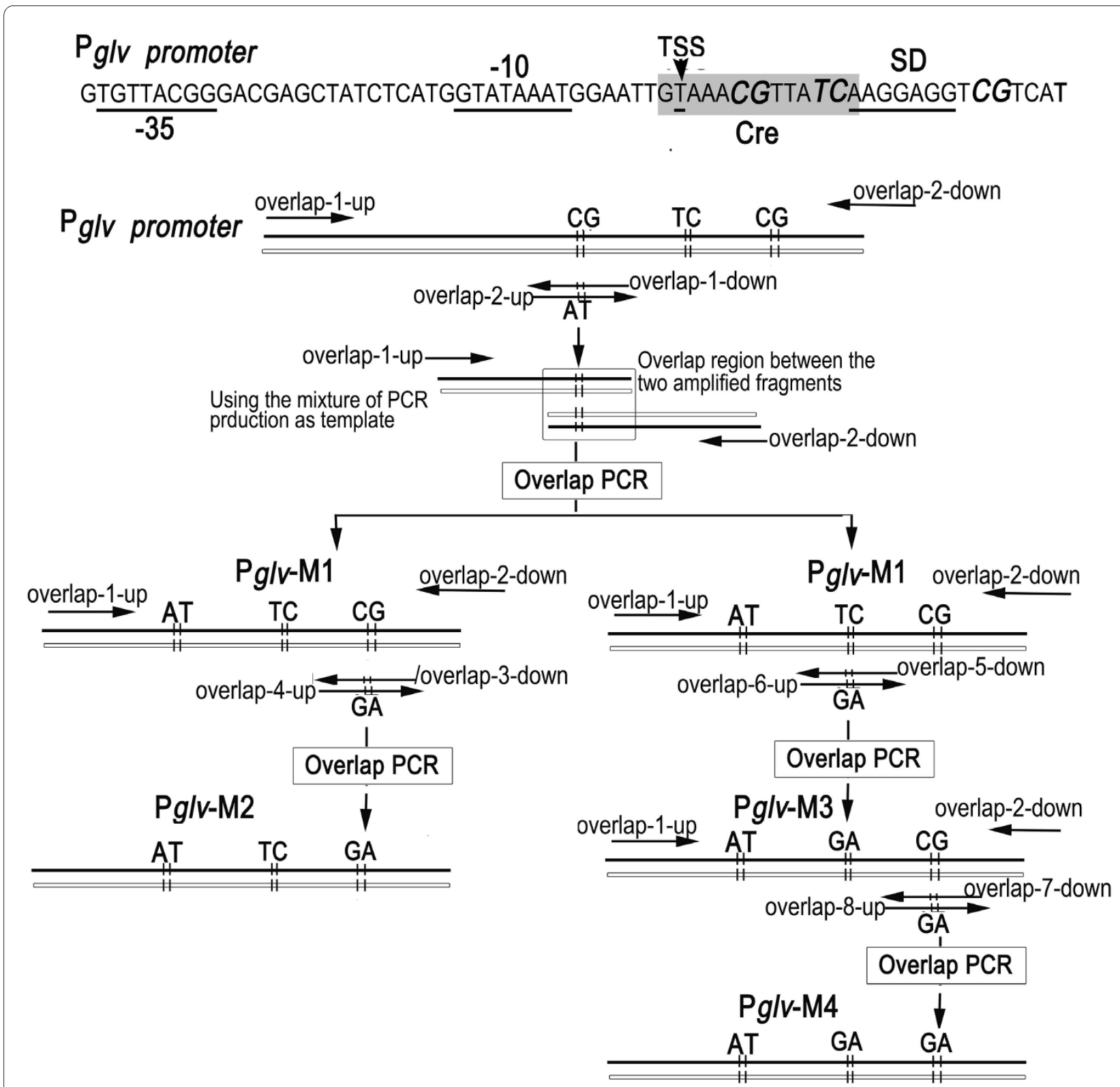

Figure 4 Schematic diagram of the overlap PCR. -10,-35, TSS, SD, and Cre represent the -10 box, -35 box, transcription origin, SD box and catabolite repression element, respectively. Italic indicates the mutated nucleotides. Approximate positions of the primers are shown with arrows. Using primer pairs overlap-1-up/overlap-1-down and overlap-2-up/overlap-2-down, a 360 bp fragment upstream of the maltose promoter and a 300 bp fragment downstream of the maltose promoter were amplified from Bacillus subtilis 1A747 genome DNA, in which there are 30 bp overlap in the two amplified fragments and the two mutant sites were introduced by primers overlap-1-down and overlap-2-up. With overlap-1-up and overlap-2-down as primers, and the mixture of two obtained fragments as template, a $630 \mathrm{bp} \mathrm{P}_{\mathrm{g} / \mathrm{v}}-\mathrm{M} 1$ was overlap PCR amplified. Same mechanism was used to generate the other three mutants $\mathrm{P}_{g / v}-\mathrm{M} 2, \mathrm{P}_{g / v}-\mathrm{M} 3$ and $\mathrm{P}_{g / v}-\mathrm{M} 4$. 
fragments as template, a $630 \mathrm{bp} \mathrm{P}_{g l v}-\mathrm{M} 1$ was overlap PCR amplified.

According to the abovementioned protocol, using $\mathrm{P}_{g l v^{-}}$ $\mathrm{M} 1$ as template, the second mutant $\mathrm{P}_{g l v}-\mathrm{M} 2$ and third mutant $\mathrm{P}_{g l v}$-M3 were amplified via overlap PCR by using of primer pairs overlap-1-up/overlap-3-down and overlap-4-up/overlap-2-down, and overlap-1-up/overlap-5down and overlap-6-up/overlap-2-down, respectively. The mutant was introduced by overlap section of overlap-3-down and overlap-4-up for $\mathrm{P}_{g l v}-\mathrm{M} 2$ and, overlap-5down and overlap-6-up for $\mathrm{P}_{g l v}$-M3. Then, the fourth mutant $\mathrm{P}_{g l v}-\mathrm{M} 4$ was generated from $\mathrm{P}_{g l v}-\mathrm{M} 3$ using primer pairs overlap-1-up/overlap-7-down and overlap-8-up/ overlap-2-down, in which the overlap section of overlap7-down and overlap-8-up introduced the mutant.

\section{Construction of plasmid vectors}

Using primer pair bga-up/bga-down, the bgaB coding for thermostable $\beta$-Gal was polymerase chain reaction (PCR) amplified from plasmid pDL. The obtained $2.0 \mathrm{~kb}$ fragment was digested with EcoRI and SacI, and cloned into pGJ103 digested with the same enzymes, resulting in pLJ2 (Figure 5).

Using Pglv-1-up and Pglv-5-down as primers, $\mathrm{P}_{g l v} \mathrm{Ma}$, $\mathrm{P}_{g l v} \mathrm{Mb}, \mathrm{P}_{g l v} \mathrm{Mc}$ and $\mathrm{P}_{g l v} \mathrm{Md}$ were PCR amplified from $\mathrm{P}_{g l v}{ }^{-}$ $\mathrm{M} 1, \mathrm{P}_{g l v}-\mathrm{M} 2, \mathrm{P}_{g l v}-\mathrm{M} 3$ and $\mathrm{P}_{g l v}-\mathrm{M} 4$ (As shown in Figure 4), respectively. After digested with $A p a \mathrm{I}$ and $\mathrm{BamHI}$, these amplified fragments were cloned into the corresponding sites of pBluskm, resulting in pJR1, pJR2, pJR3 and pJR4. A ribosome binding site (RBS) fragment INSD generated

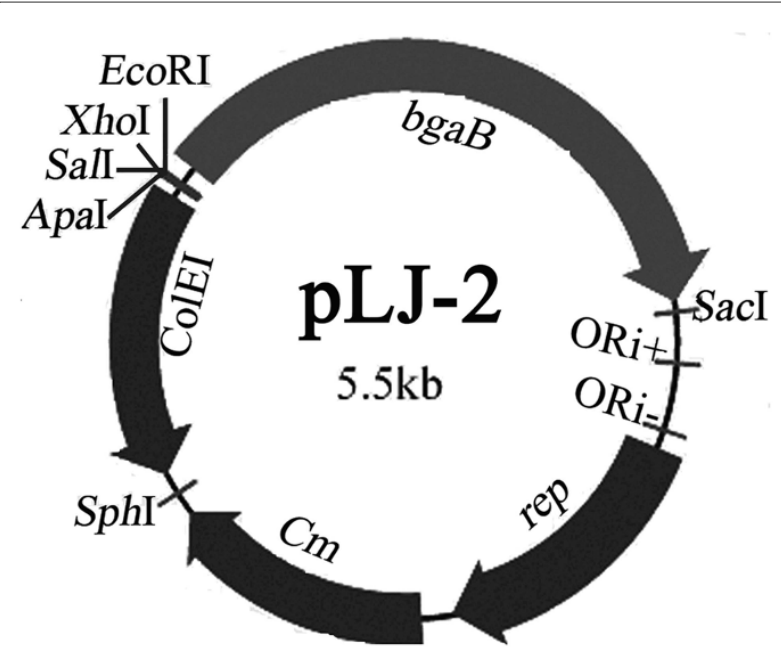

Figure 5 Map of the promoter probe vector pLJ-2. ORI+, ORI- and rep represent the single-strand replication origin, the double strand origin and replication protein in $\boldsymbol{B}$. subtilis, respectively. ColEl, bgaB and $\mathrm{Cm}$ represent $E$. coli $\mathrm{ColEl}$ replicon, chloramphenicol-resistance marker and coding gene of $\beta$-Gal. The unique restriction sites are marked on the outside of the map. by annealing two artificially synthesized oligonucleotides INSID-1 and INSID-2, was cloned into the pJR1-4 digested by $B a m \mathrm{HI}$ and $S a c \mathrm{I}$, respectively. Then, the four mutant promoters with engineered RBS were excised from the obtained four recombinants with ApaI and EcoRI, and cloned into the corresponding sites of pLJ-2, in which the $\operatorname{bgaB}$ was used as reporter, yielding pJRINM1, pJRINM2, pJRINM3 and pJRINM4, respectively.

\section{Reconstruction of $B$. subtilis strain}

With B. subtilis $1 \mathrm{~A} 747$ chromosomal DNA as template, the two homogeneous arms GAf (using GlvA-fro-up and GlvA-fro-down as primers) and GAb (using GlvA-bac-up and GlvA-bac-down as primers) were amplified. A selection marker (spectinomycin resistance gene) was generated through PCR amplified from plasmid pDG1728, using primers Spec-I-up and Spec-I-down. And BamHISalI treated selection marker and KpnI-ApaI treated GAf was cloned into pBluskm, resulting in pYG34.

The expression cassette directed by $\mathrm{P} 43$ promoter was constructed as follows. First, the P43 promoter [3,17] was amplified from B. subtilis $1 \mathrm{~A} 747$ chromosomal DNA, using primers P43-1-up and P43-1-down. After digestion with ApaI and EcoRI, the P43 promoter was cloned into the corresponding sites in pBluskm, yielding $\mathrm{pB} 43$. Then the GAb fragment, digested with EcoRI and SacI, was cloned into the corresponding sites in $\mathrm{pB} 43$, resulting in pCYL17. Finally, with primers P43-2-up and GlvA-bacdown, the P43 promoter and the GAb were amplified as a single product from pCYL17 and ligated with pYG34 after digestion with $B a m \mathrm{HI}$ and SacI, resulting in pCYL25. The B. subtilis 1 A747 was transformed with the linear fragment of pCYL25 obtained with ScaI to replace promoter Pglv with P43 via a double crossover event between the linearized PCYL25 and B. subtilis 1A747 chromosome. The spectinomycin resistance $\left(\mathrm{Spec}^{\mathrm{R}}\right)$ colonies were selected. And the resultant strain was named as BCYL.

\section{Isolation of total RNA and Real-time PCR}

The cultures was harvested at $24 \mathrm{~h}$. Total RNA of bacteria was isolated by using SV total RNA isolation kit (Cat. Z3100, Promega). The cDNA chain was synthesized by using Reverse Transcription System (Cat. A3500, Promega).

And Real-time PCR was performed by using Real time PCR Kit (Cat. DRR041 S, TaKaRa). The bagB gene was amplified using bga-up and bga-down as primers B1-up/ B1-down. With the primers 16s-up/16s-down, Bacillus subtilis $16 \mathrm{~s}$ rDNA was amplified as control, The PCR protocol was as follows: $2 \mathrm{~min}$ at $50^{\circ} \mathrm{C}, 10 \mathrm{~min}$ at $95^{\circ} \mathrm{C}$, and then 35 cycles consisting of $45 \mathrm{~s}$ at $95^{\circ} \mathrm{C}, 1 \mathrm{~min}$ at 
$52^{\circ} \mathrm{C}$, and $30 \mathrm{~s}$ at $72^{\circ} \mathrm{C}$. Reactions were carried out in realtime PCR detection system (IQ5, Bio-RAD).

\section{SDS-PAGE assay}

Sodium dodecylsulfate polyacrylamide gel electrophoresis (SDS-PAGE) was performed as described previously [14].

\section{$\beta$-Gal activity assay}

Method has been described previously [[11,18] and [19]]. In brief, the culture was pelleted by centrifuge and resuspended in an equivalent volume of buffer $Z[18,19]$. Using buffer Z, 0.01 or $0.1 \mathrm{~mL}$ sample aliquots was diluted to 0.8 $\mathrm{mL}$, and then added $0.01 \mathrm{~mL}$ of lysozyme stock $(10 \mathrm{mg} /$ $\mathrm{ml}$ ). The mixture was incubated at $37^{\circ} \mathrm{C}$ for $30 \mathrm{~min}$, and then, added $0.2 \mathrm{ml}$ of $4 \mathrm{mg} / \mathrm{ml}$ o-nitrophenyl-b-Dgalactopyranoside (ONPG). After incubated at $55^{\circ} \mathrm{C}$ for $15 \mathrm{~min}$, the reaction was stopped by adding $0.5 \mathrm{ml}$ of $1 \mathrm{M}$ $\mathrm{Na}_{2} \mathrm{CO}_{3}$. Absorbance was recorded at $420 \mathrm{~nm}$ with a spectrophotometer (HITACHI, U-3010). One unit of $\beta$ galactosidase activity was defined as the amount of enzyme necessary to release $1 \mu \mathrm{mol} 2$-nitrophenol from o-nitrophenylgalactopyranoside per minute at $55^{\circ} \mathrm{C}$. $\beta$ galactosidase activity is expressed as units per $\mathrm{mL}$ sample.

\section{Southern blot analysis}

Southern blot analysis of chromosomal DNA digested with ApaI and EcoRI was carried out as described previously [14]. Probe labeling was performed with a DIG DNA labeling kit (Roche, Cat.No.1093657) according to the instructions, using the spectinomycin resistance gene as template.

\section{Reducing sugar assay}

Reducing sugar was measured by the dinitrosalicylic method (DNS) [20] using glucose as a standard.

\section{Assay of glucose concentration}

The concentration of glucose was determinate as previously described [21]

\section{Additional material}

Additional file 1 The growth curves from B. subtilis 1 A747 harboring different plasmids when cultured in LB. (black diamond) represents OD595 from B. subtilis 1 A747 harboring pLJ-7; (black square) represents OD595 from B. subtilis 1 A747 harboring pJRINM1; (black triangle) represents OD595 from B. subtilis 1 A747 harboring pJRINM2; cross $(x)$ represents OD595 from B. subtilis 1 A747 harboring pJRINM3; asterisk $\left(^{*}\right)$ represents OD595 from B. subtilis 1 A747 harboring pJRINM4.

Additional file 2 The growth curves from B. subtilis 1 A747 harboring different plasmids when cultured in LB supplemented with $5 \%$ maltose. (black diamond) represents OD595 from B. subtilis 1A747 harboring pL-7; (black square) represents OD595 from B. subtilis 1A747 harboring pJRINM1; (black triangle) represents OD595 from B. subtilis 1 A747 harboring pJRINM2; cross $(X)$ represents OD595 from B. subtilis 1 A747 harboring pJRINM3; asterisk $(*)$ represents OD595 from B. subtilis 1A747 harboring pJRINM4
Additional file 3 The growth curves from B. subtilis $1 \mathrm{~A} 747$ harboring different plasmids when cultured in LB supplemented with $5 \%$ maltose plus $\mathbf{5 \%}$ glucose. (black diamond) represents OD595 from B. subtilis 1 A747 harboring pLJ-7; (black square) represents OD595 from B. subtilis 1 A747 harboring pJRINM1; (black triangle) represents OD595 from B. subtilis 1 A747 harboring pJRINM2; cross $(X)$ represents OD595 from B. subtilis 1 A747 harboring pJRINM3; asterisk (*) represents OD595 from B. subtilis 1 A747 harboring pJRINM4.

Additional file 4 Plasmids used in this study

Additional file 5 Primers and oligonucletides used in this study

Competing interests

The authors declare that they have no competing interests.

\section{Authors' contributions}

MMY initiated and coordinated the project. WWZ and MMY were responsible for site-directed mutagenesis and its analysis. WWZ and YLC performed the batch cultivation. MMY and Y-S Gong performed construction of expression system. All authors wrote the paper and approved the final version of the manuscript.

\section{Acknowledgements}

This work was supported by grants from Chinese National Natural Science Foundation (30871813). And we thank the Bacillus Genetic Stock Centre of Ohio State University for generously providing the study materials.

\section{Author Details}

College of Animal Science and Technology, Northwest A\&F University, Yangling 712100, China

Received: 26 February 2010 Accepted: 10 July 2010

Published: 10 July 2010

\section{References}

1. Kunst F, Ogasawara N, Moszer I, Albertini AM, Alloni G, Azevedo V, Bertero MG, Bessieres P, Bolotin A, Borchert S: The complete genome sequence of the gram-positive bacterium Bacillus subtilis. Nature 1997, 390:249-256.

2. Schallmey M, Singh A, Ward OP: Developments in the use of Bacillus species for industrial production. Can J Microbiol 2004, 50:1-17.

3. Zhang XZ, Cui ZL, Hong Q, Li SP: High-level expression and secretion of methyl parathion hydrolase in Bacillus subtilis WB800. Appl Environ Microbiol 2005, 71:4101-4103.

4. Zhang AL, Liu H, Yang MM, Gong YS, Chen H: Assay and characterization of a strong promoter element from B. subtilis. Biochem Biophys Res Commun 2007, 354:90-95.

5. Nguyen HD, Nguyen QA, Ferreira RC, Ferreira LCS, Tran LT, Schumann W: Construction of plasmid-based expression vectors for Bacillus subtilis exhibiting full structural stability. Plasmid 2005, 54:241-248.

6. Nguyen HD, Schumann W: Novel plasmid-based expression vectors for intra- and extracellular production of recombinant proteins in Bacillus subtilis. Prot Expr Purif 2006, 46:189-195.

7. Li W, Li HX, Ji SY, Li S, Yang MM, Chen YL: Characterization of two temperature-inducible promoters newly isolated from $B$. subtilis. Biochem Biophys Res Commun 2007, 358:1148-1153.

8. Kim L, Mogk A, Schumann W: A xylose-inducible Bacillus subtilis integration vector and its application. Gene 1996, 87:53-61.

9. Bhavsar AP, Zhao XM, Brown ED: Development and characterization of a xylose-dependent system for cloned gene in Bacillus subtilis: conditional complementation of a Teichoic acid mutant. Appl Environ Microbiol 2001, 67:403-410.

10. Hartl B, Wehrl W, Wiegert T, Homuth G, Schumann W: Development of a new integration site within the Bacillus subtilis chromosome and construction of compatible expression cassettes. J Bacterio/ 2001, 183:2696-2699.

11. Yang MM, Zhang WW, Zhang XF, Cen PL: Construction and characterization of a novel maltose inducible expression vector in Bacillus subtilis. Biotechnol Lett 2006, 28:1713-1718.

12. Thompson J, Pikis A, Ruvinov SB, Henrissat B, Yamamoto H, Sekiguchi J: The gene g/vA of Bacillus subtilis 168 encodes a metal-requiring NADHdependent 6-phospho-glucosidase. J Bol Chem 1998, 273:27347-27356. 
13. Yamamoto H, Serizawa M, Thompson J, Sekiguchi J: Regulation of glvoperon in Bacillus subtilis: YfiA(GLVR) is a positive regulator of the operon that is repressed through CcpA and cre. J Bacteriol 2001, 183:5110-5121

14. Sambrook J, Fitsch EF, Maniatis T: Molecular Cloning: A Laboratory Manual Cold Spring Harbor: Cold Spring Harbor Press; 1989.

15. Bron S: Plasmids. In Molecular biological methods for Bacillus Edited by: Harwood CR, Cutting SM. New York: John Wiley and Sons; 1990:75-174

16. Yang MM, Zhang WW, Bai XT, Li HX, Cen PL: Electroporation is a feasible method to introduce circularized or linearized DNA into $B$. subtilis chromosome. Mol Biol Rep 2010 in press.

17. Wang $P Z$, Doi RH: Overlapping promoters transcribed by Bacillus subtilis $\sigma^{55}$ and $\sigma^{37}$ RNA polymerase holoenzymes during growth and stationary phase. J Biol Chem 1984, 259:8619-8625.

18. Hirata H, Fukazawa T, Negoro S, Okada H: Structure of a $\beta$-galactosidase gene of Bacillus stearothermophilus. J Bacteriol 1986, 166:722-727.

19. Fanning S, Leahy M, Sjheehan D: Nucleotide and deduced amino acidsequences of Rhizobium meliloti 102F34 lacZ gene: comparison with prokaryotic $\beta$-galactosidases and hunan $\beta$-glucuronidase. Gene 1994, 141:91-96.

20. Miller GL: Use of dinitrosalicylic acid reagent for determination of reducing sugar. Anal Chem 1959, 31:426-428.

21. Cavari BZ, Phelps G: Sensitive Enzymatic Assay for Glucose Determination in Natural Waters. Appl Environ Microbiol 1977 33:1237-1243

22. Short JM, Fernandez JM, Sorge JA, Huse WD: $\lambda$ ZAP: A bacteriophage $\lambda$ expression vector with in vivo excision properties. Nucleic Acids Res 1988, 16:7583-7600

23. Guerot-Fleury AM, Frandsen N, Stragier P: Plasmids for ectopic integration in Bacillus subtilis. Gene 1996, 180:57-61.

doi: 10.1186/1475-2859-9-55

Cite this article as: Ming et al., Development of a Bacillus subtilis expression system using the improved Pg/v promoter Microbial Cell Factories 2010, 9:55

Submit your next manuscript to BioMed Centra and take full advantage of:

- Convenient online submission

- Thorough peer review

- No space constraints or color figure charges

- Immediate publication on acceptance

- Inclusion in PubMed, CAS, Scopus and Google Scholar

- Research which is freely available for redistribution

Submit your manuscript at www.biomedcentral.com/submit
C Biomed Central 